\title{
Schätzung inzuchtwirksamer (effektiver ) Populationsgrößen aus Genfrequenzschwankungen bei Bayerischem Fleckvieh und Tiroler Grauvieh
}

\begin{abstract}
Summary
Title of the paper: Estimating effective population size (Ne) from allele frequency changes in Bavarian Simmental (FV) and Tyrolean Grey (GV) cattle

Frequencies of 10 to 16 alleles of blood group, hemo- and lactoprotein loci were estimated for FV in 1960, 1986 and 200, for GV in 1969, 1979 and 1998. Rates of inbreeding in the intervening periods and Ne`s were derived from Wahlund variances. The Ne`s for the two part periods of FV, spanning 2.2 resp. 4.1 generations, were 152 resp. 147, for the whole period of 6.25 generation intervals 373 . This is due to the opposing signs of the changes in the two part periods and it may reflect the large influence of a top sire on the gene pool in the first period and ensueing change to a FV gene reservoir similar to that in 1960. For GV the Ne of the two part periods were 139 and 56, for the whole 97, roughly in agreement with the one expected from the two short periods. The lower Ne of the second part period agrees well with an estimate by the inbreeding increment in roughly the same time period.
\end{abstract}

Key Words: inbreeding increment, effecive population size, Wahlund variance, continental cattle

\section{Zusammenfassung}

Wahlund Varianzen, Inzuchtzuwachsraten und effektive Populationsgrößen (Ne) wurden aus Frequenzschwankungen von 10 bis 16 Allelen von Blutgruppen-, Hämo- und Laktoproteinloci bei Bayerischem Fleckvieh (FV) und Tiroler Grauvieh (GV) geschätzt. Die hierfür bei FV genommenen Blutproben stammten aus der Zeit um 1960, 1986 und 2000, die vom GV aus 1969, 1979 und 1998. Ne für die 4,1 Generationsintervalle umfassende erste Periode bei FV war 152, für die zweite 2,2 Generationsintervalle lange 147, für die Gesamtperiode von 6,25 Generationsintervallen aber 373. Die Frequenzschwankungen zwischen 1960 und 2000 war offensichtlich geringer als die in den Teilperioden, zurückzuführen darauf, dass in diesen die Veränderungen entgegengesetzte Richtungen hatten. Abgesehen von Zufall, könnte dies die starke Präsenz der Gene des Spitzenvererbers Haxl in der ersten Teilperiode und deren Austausch gegen solche des früher vorhandenen Genpools reflektieren. Bei GV waren die Ne Werte der beiden Teilperioden 139 und 56, für die Gesamtperiode 97, nicht weit von dem aus den Ne's erwarteten Wert. Der geringere Wert der zweiten Teilperiode entspricht in etwa der aus der Inzuchtzuwachsrate für GV im selben Zeitraum geschätzten $\mathrm{Ne}$.

Schlüsselwörter: Inzuchtzuwachs, effektive Populationsgröße, Wahlund Varianz, mitteleuropäische Rinderrassen

Der Erhaltung genetischer Vielfalt wird in der letzten Zeit vermehrt Aufmerksamkeit geschenkt, vor allem in Hinblick auf gefährdete Rassen, doch wird auch die mögliche Verengung des Erbmaterials in Leistungsrassen, wie sie sich in gesteigerten Inzuchtzuwachsraten ausdrückt, beachtet. MEUWISSEN und WOOLLIAMS (1994) untersuchten die inzuchtwirksame (effektive) Populationsgröße, welche Fitness Minderung vermeidet, GIBSON und KENNEDY (1990) prüften restringierte Selektionsindices, die Verminderung von selektionsbedingtem Verwandtschaftszuwachs ermöglichen sollen. GRUNDY et al. (1994) schlagen Verwendung verzerrter Parameter mit ähnlicher Zielsetzung vor. Die besondere Bedeutung von Selektionsschärfe bei BLUP Selektion in Hinblick auf Steigerung von Verwandtschaftszuwächsen wird von BIJEMA und WOOLLIAMS (2000) hervorgehoben. Den Einfluss verschiedener Selektions- 
strategien auf Inzuchtzuwachs bzw. Selektionserfolg haben anhand von Simulationsstudien SENOU (1990) und speziell bei Schweinenukleusherden SCHÜTTE et al. (2002) untersucht.

Die klassische Methode zur Untersuchung des Verwandtschaftszuwachses ist die Pedigree Analyse, die von Sewall WRIGHT eingeführt wurde. Ihre Anwendung bei mitteleuropäischen Rinderrassen wird allerdings durch oft unvollständige Abstammungsaufzeichnungen bei noch oder jedenfalls bis vor kurzem üblichen offenen Herdbüchern erschwert. Wo diese geschlossen sind, wie bei amerikanischen oder auf diese direkt zurückgehende Populationen (CASANOVA et al., 1992; TEBRAKE et al. 1994) oder bei einheimischen Pferderassen etwa, kann die Schätzung der effektiven Populationsgröße (Ne) natürlich auf diese Weise vorgenommen werden (FEHLINGS et al., 1983). SÖLKNER et al. (1998) haben z.B. gefunden, dass in der vierten Ahnengeneration bei österreichischem Fleckvieh weniger als 2/3 der Pedigrees noch vollständig waren, bei Grauvieh allerdings noch fast $90 \%$.

Auf die Schätzung von Inzuchtzuwachs aus der Populationsgröße hat Sewall WRIGHT aufmerksam gemacht. Die von ihm vorgeschlagene Formel setzt Panmixie etc. voraus. LATTER (1959) hat sie für die Hierarchie strukturierter Haustierpopulationen modifiziert, aber WRAY und THOMPSON (1990) haben gezeigt, dass auch damit der Inzuchtzuwachs bei vorhandener Selektion unterschätzt wird.

Ne kann aber aus dem Umfang zufallsbedingter Frequenzschwankungen neutraler Gene geschätzt werden , die weitgehend zu denselben Ergebnissen ,wie die Schätzung aus Inzucht- oder Verwandtschaftszuwachs führen.(CROW und KIMURA, 1970). Die Vorgangsweise bei der Schätzung von Ne aus zeitlich aufeinander folgenden Schwankungen von Genhäufigkeiten haben u.a. der Autor (PIRCHNER, 1983), GRAML et al. (1988) und WAPLES (1989) dargestellt und POLLAK (1983) hat sie erläutert und zusammengefasst.

In der vorliegenden Arbeit werden Marker Genfrequenzen, vor allem von Blutgruppen, aber auch von Hämo- und Laktoproteinen in Hinblick auf im Ablauf von bis zu 40 Jahren erfolgten, als zufällig betrachtete Schwankungen bei ihnen, untersucht. Aus den gefundenen Schwankungen werden die inzuchtwirksamen (effektiven) Populationsgrößen bei Bayerischem Fleckvieh (FL) und Tiroler Grauvieh (GV) geschätzt.

\section{Material und Methoden}

Die zufallsbedingten Änderungen der Genhäufigkeiten wurden vorwiegend bei Blutgruppen und teilweise auch bei Transferrin und Laktoproteinen untersucht. Die Häufigkeiten vom Fleckvieh stammen von BUSCHMANN (1962), HIERL et al. (1978) und von LANG (1987), Milchprotein von MEYER (1967), Laktoproteingenhäufigkeiten von GRAML et al. (1984) und jene für die Populationswerte des Jahres 2000 wurden vom Blutgruppenlabor Grub (HERTNER, 2001) zur Verfügung gestellt. Die Genfrequenzen des Tiroler Grauviehs sind für 1969 von ERLACHER (1970) publiziert worden, die späteren wurden von den Zuchtverbänden für Nord- und für Südtirol zur Verfügung gestellt (HAUSEGGER, 1979, 1998).

Je Locus wurde ein Allel genommen, bei Loci A und SH und bei Transferrinen die zwei bzw. drei häufigsten.

Der Schätzwert der Wahlund Varianz (LOPEZ-FANJUL, 1974; HATTEMER, 1982) wird für Stichprobenvariation korrigiert 


$$
\mathrm{Vw}=\left((\mathrm{p} 1-\mathrm{p} 2)^{2}-\mathrm{s}^{2} 1-\mathrm{s}^{2} 2\right) / \mathrm{p}(1-\mathrm{p})
$$

p1, p2 symbolisieren die Genfrequenzen von Kohorte (Jahrgang bzw. Zeitabschnitt)1 bzw. $2, p=(p 1+p 2) / 2, s^{2} 1$ bzw. $s^{2} 2=p q / 2 N$ oder $(1-q)^{2} / 4 N, N$ ist die Stichprobengröße.

Die Schätzung des Verwandtschafts- bzw. Inzuchtzuwachses erfolgt aus

$$
\mathrm{F}=(1-(1-1 / 2 \mathrm{Ne}))
$$

$\mathrm{Ne}=1 / 2 \mathrm{dF}, \mathrm{dF}$ ist der Inzuchtzuwachs je Generation.

Der 95\% Vertrauensbereich für $F$ resultiert aus

$\mathrm{nF} / \mathrm{X}^{2}(\mathrm{a} / 2)(\mathrm{n})$ bzw. nF/X²(1-a/2)(n) wobei $n$ die Summe der Freiheitsgrade, für jeden Locus (Allelzahl-1), symbolisiert; $\mathrm{a} / 2=.025$.

Das Generationsintervall für Bayerisches Fleckvieh (FV) wurde mit 6,4 Jahren angenommen (KERLER, 1987), das für Tiroler Grauvieh (GV) mit 5 Jahren, da im Gegensatz zu Fleckvieh bei diesem in stärkerem Maß Jungstiere verwendet werden.

\section{Ergebnisse und Diskussion}

In Tabelle 1 werden Genhäufigkeiten, in Tabelle 2 Wahlund Varianzen für die einzelnen Loci bei Fleckvieh und Grauvieh angegeben. Inzuchtzuwachsraten und Ne sind in Tabelle 3 angeführt. Für die Teilperioden 1960 - 1986 und 1986 - 2000 hat FV Ne Werte von 134 und 128 bzw. wenn Laktoproteine einbezogen werden, von 152 und 147. Wenn die zwei negativen Werte der zweiten Kohorte mit Null angenommen werden, ist Ne 118 bzw. mit Laktoproteinen 135. Für die ganze 6,25 Generationen umfassende Periode von 1960 bis 2000 ergibt die Schätzung eine Ne von 323 ohne und 373 mit Laktoproteinen und von 294 bzw. 291 wenn die negativen Wahlund Varianzen ignoriert werden.

Für Grauvieh sind die effektiven Populationsgrößen für die nur zwei Rindergenerationen umfassende erste Teilperiode 139, für die zweite nahezu vier Generationen umfassende 56,5 und für den gesamten Zeitraum 97,2.

Die Gene werden als neutral angenommen, jedenfalls in Hinblick auf die relativ kurzen Zeitperioden. Im Falle der Laktoproteine ist das mit mehr Einschränkungen zu betrachten als bei Blutgruppen. Jedenfalls wird Selektion die zufällige Schwankung der Häufigkeiten mindern und diese eher in der Nähe der Gleichgewichtsfrequenz halten. Die höheren Ne Werte bei Einbeziehung der Laktoproteine können darauf zurückgeführt werden.

Die über den ganzen Zeitraum geschätzte inzuchtwirksame Populationsgröße würde als ungefähres Mittel der beiden Teilperioden erwartet werden. Die erwartete Heterozygotieminderung über die Gesamtperiode ergibt sich aus dem Produkt der beiden Teilperioden und würde für Fleckvieh zu einer Ne von 142,2, bei Grauvieh zu einer von 71 führen. Die aus der relativ geringen Veränderung der Genhäufigkeiten in der Periode von 1960 bis 2000 bei Fleckvieh auf Ne von 373 geschätzte ist aber wesentlich größer und überschreitet den 97,5\% Vertrauensbereich beider Teilperioden. Bei Grauvieh liegt der Ne Wert der Gesamtperiode zwischen denen der beiden Teilperioden und innerhalb der betreffenden Vertrauensbereiche.

Womit kann die hohe Ne der Gesamtperiode bei Fleckvieh erklärt werden, bzw. was kann die Ursache sein, abgesehen davon, dass die Vertrauensbereiche der Schätzungen von den drei Kohorten sich überlappen? Die Differenzen zwischen Genfrequenzen 
Tabelle 1

Häufigkeiten von Blutgruppen, Hämo- und Laktoproteingenen bei Bayerischem Fleckvieh und Tiroler Grauvieh (Frequencies of bloodgroup, hemo- and lactoprotein genes in Bavarian Fleckvieh (Bavarian Simmental) and Tyrolean Grey)

\begin{tabular}{|c|c|c|c|}
\hline & $\begin{array}{l}\text { Genfrequenzen bei Fleckvieh } \\
\text { Blutgruppen }\end{array}$ & & \\
\hline & 1960 & 1986 & 2000 \\
\hline $\mathrm{n}$ & 1000 & 1040 & 198 \\
\hline \multicolumn{4}{|l|}{ Allel } \\
\hline $\mathrm{a}$ & .572 & .647 & .626 \\
\hline A1 & .176 & .207 & .165 \\
\hline $\mathrm{A} 1 \mathrm{H}$ & .242 & .146 & .172 \\
\hline F & .770 & .826 & .768 \\
\hline j & .899 & .880 & .871 \\
\hline $\mathrm{l}$ & .850 & .868 & .888 \\
\hline M & .983 & .991 & .995 \\
\hline $\mathrm{R}$ & .165 & .227 & .146 \\
\hline $\mathrm{Z}$ & .620 & .540 & .508 \\
\hline S'H & .267 & .359 & .296 \\
\hline $\mathrm{s}$ & & .086 & .171 \\
\hline H’ & & .295 & .288 \\
\hline H'H', & & .092 & .110 \\
\hline U’ & & .144 & .084 \\
\hline \multicolumn{4}{|c|}{ Laktoproteine } \\
\hline & 1966 & 1978 & 1994 \\
\hline $\mathrm{n}$ & 276 & 2262 & 3902 \\
\hline CASAS1 & .871 & .900 & .898 \\
\hline LGB & .499 & .482 & .485 \\
\hline \multicolumn{4}{|c|}{ Genfrequenzen bei Grauvieh } \\
\hline & 1969 & 1979 & 1998 \\
\hline $\mathrm{n}$ & 401 & 389 & 110 \\
\hline $\mathrm{b}$ & .849 & .846 & .745 \\
\hline $\mathrm{B}$ & .075 & .101 & .073 \\
\hline G & .015 & .0025 & .045 \\
\hline $\mathrm{F}$ & .870 & .825 & .973 \\
\hline I & .170 & .260 & .309 \\
\hline $\mathrm{R}$ & .240 & .280 & .409 \\
\hline Z & .693 & .707 & .688 \\
\hline $\mathrm{HBB}$ & .840 & .780 & .773 \\
\hline Am & .670 & .620 & \\
\hline TF A & .180 & .160 & .183 \\
\hline $\mathrm{D}$ & .290 & .220 & .349 \\
\hline $\mathrm{E}$ & .520 & .560 & .445 \\
\hline $\mathrm{a}$ & .340 & .350 & \\
\hline $\mathrm{Pt}$ & & .776 & $.684^{*}$ \\
\hline
\end{tabular}

aufeinander folgender Kohorten sind größer als die zwischen 1960 und 2000. Das ist formal darauf zurückzuführen, dass die Richtungen der Differenzen in der zweiten Periode entgegengesetzt der Richtung in der ersten sind, die Korrelation zwischen den Frequenzdifferenzen in der ersten und der zweiten Periode ist auch hochsignifikant negativ.

Offensichtlich haben sich die Häufigkeiten der neutralen Gene der letzten (2000) Kohorte wieder den Ausgangshäufigkeiten genähert, während die der dazwischen liegenden sich davon entfernt hatten. Unter der Annahme neutraler Gene ist das der Drift zuzuschreiben. Von Bedeutung könnte in diesem Zusammenhang aber sein, dass sich hier der überragende Einfluss des Stieres Haxl manifestiert. KERLER (1987) fand, dass etwa 16\% der Gene des Testbullenjahrganges 1983/84 von dem damaligen 
Spitzenbullen Haxl stammten und diese Gruppe oder nahe Verwandte haben sicher einen erheblichen Teil der in der Stichprobe 1986 vorhandenen Blutproben beigesteuert. Zur Erklärung müsste dann allerdings die Kohorte 2000 von Tieren sein, die in ihren (neutralen) Genfrequenzen sich wieder denen der Population von 1960 angenähert haben. Über ein ähnlich schwierig zu erklärendes Ergebnis, allerdings von einer Wildpopulation von Fischen, berichten MILLER und KAPUSCINSKI (1997) und interpretieren es ebenfalls mit genetischer Drift zurück zur Ausgangspopulation.

Tabelle 2

Wahlundvarianzen (Wahlund variances)

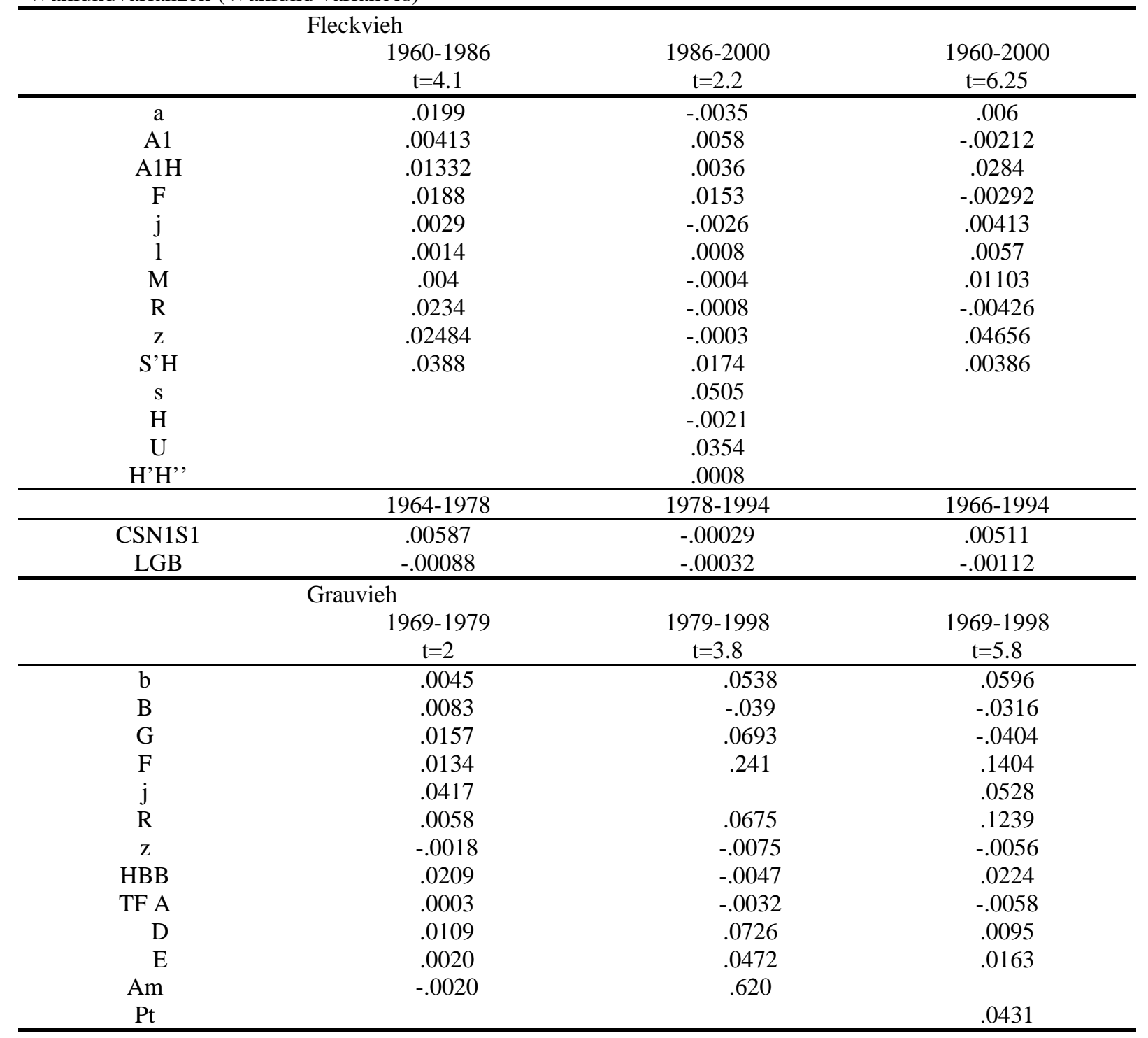

KERLER (1987) hat versucht, aus den Abstammungsaufzeichnungen Inzuchtzuwachs beim Bayerischen Fleckvieh zu schätzen, konnte aber wegen der Lückenhaftigkeit der Vorfahreninformation zu keinem akzeptablen Resultat kommen. REINHARDT (1986) hat für Testbullen der Jahre 1979-1985 die Inzuchtkoeffizienten geschätzt. Der Anstieg zwischen den Jahrgängen 1979/80 und 1984/85 beträgt ,0025, was ,wenn das als Generationsintervall angenommen wird, zu einer Ne von 200 führt. KERLER (1987) hat aus der Struktur der Bayerischen Fleckviehpopulation nach der von LATTER (1959) vorgeschlagenen und von HILL (1979) weiter modifizierten Formel die wirksame Ne auf etwa 800 geschätzt. Die Berücksichtigung des Selektionseinflusses, 
wie sie WRAY und THOMPSON (1990) vorgeschlagen haben, verlangt Multiplikation der Differenz zwischen Inzuchtzuwachs, geschätzt nach LATTER (1959) und der bei Zufallspaarung erwarteten, mit einem von der Selektionsmethode abhängigen Koeffizienten, der bei Verwendung von Verwandteninformation höher ist als bei Individualselektion. Dieser kann für die bei Fleckvieh praktizierte Selektion mit 2,5 angenommen werden, was dann zu einem Inzuchtzuwachs von etwa 0,002 und $\mathrm{Ne}$ von 250 führt, in den Bereich des aus Gendrift geschätzten Wertes.

Tabelle 3

Inzucht-(drift)-wirksame Populationsgrößen (Ne) bei Bayerischem Fleckvieh (FV) und Tiroler Grauvieh (GV) (Effective population size (Ne) of Bavarian Fleckvieh (FV) and Tyrolean Grey (GV))

\begin{tabular}{|c|c|c|c|c|c|c|c|}
\hline & & & FG & $\mathrm{dF}$ & $\mathrm{Ne}$ & $\begin{array}{c}\text { untere } \\
\text { Vertrauensg. }\end{array}$ & obere \\
\hline \multirow[t]{6}{*}{ FV } & 1960-1986 & ohne LP & 10 & .0037 & 134 & & \\
\hline & & mit & 12 & .0033 & 152 & 55 & 297 \\
\hline & 1986-2000 & ohne LP & 14 & .0039 & 128 (118) & & \\
\hline & & mit & 16 & .0034 & 147 (135) & 63 & 255 \\
\hline & $1960-2000$ & ohne LP & 10 & .0016 & 323 (294) & & \\
\hline & & mit & 12 & .0013 & $152(291)$ & 135 & 732 \\
\hline \multirow[t]{3}{*}{ GV } & 1969-1979 & ohne LP & 13 & .0036 & 139 (114) & 53 & 267 \\
\hline & 1979-1998 & ohne LP & 12 & .0088 & 56 (43) & 20 & 112 \\
\hline & 1969-1998 & ohne LP & 11 & .0051 & 97 (74) & 32 & 196 \\
\hline
\end{tabular}

Generationsintervall bei FV 6.4, bei GV 5 Jahre; damit umfassen Zeitintervalle bei FV 4.1, 2.2, 6.25 Generationen, bei GV 2, 3.8 und 5.8 In Klammern Ne Werte, wo Minuswerte der Wahlund Varianz ignoriert worden sind

DF Inzuchtzuwachs je Generation; LP Laktoproteine

SÖLKNER et al. (1998) haben, allerdings nur von einer Generation, Inzuchtzuwachs bei österreichischem Fleckvieh und Grauvieh geschätzt, die zu Ne`s von 258 bzw. 73 führen. Allerdings war bei Fleckvieh etwas Einfluss von (geringgradiger) Einkreuzung vorhanden, während in dem hier untersuchten Material solche Tiere ausgeschlossen worden sind. Jedenfalls stimmt aber der von den Autoren für Grauvieh gefundene Inzuchtzuwachs sehr gut mit dem aus Genfrequenzschwankungen in der zeitlich fast identischen Periode überein.

KROGMEIER et al. (1997) zeigen Inzuchtzuwächse bei Gelbvieh und bayerischem und württembergischem Braunvieh über etwa 11 Jahre, etwa 2 Generationen. Es resultieren Zuwächse von 0,71 und 0,77 \% und Ne's von 141 bzw. 130. Aus den auch hier verwendeten Blutgruppengenfreqenzen von 1986 und einer umfangreichen Stichprobe aus 1968 haben LANG (1987) und GRAML et al. (1988) eine ähnliche Ne (192) geschätzt und für die zahlenmäßig viel kleinere Murnau-Werdenfelser Rasse über den annähernd gleichen Zeitraum eine Ne von 34.

In Kontrast zu diesen Werten stehen Inzuchtzuwachsraten von amerikanisch beeinflussten Milchrassen wie Brown Swiss und Holstein Friesian, bei denen sowohl in der Schweiz wie in Holland (aber auch in den USA) in den letzten zwei oder drei Rindergenerationen Inzuchtzuwachsraten existieren, die mit Ne von erheblich weniger als hundert verbunden sind (CASANOVA et al., 1992; TEBRAKE et al., 1994; GODDARD und WIGGANS, 1999).

Es ergibt sich die Frage, warum die Höhenrassen, insbesondere das Fleckvieh, wesentlich geringeren Verwandtschaftszuwachs und größere Ne zeigen als die beiden Milchrassen. Es bieten sich zwei einander nicht ausschließende Erklärungen an. Einmal sind es Doppelnutzungsrassen, bei welchen Milch zwar am meisten Beachtung findet, Fleischleistung aber doch nicht unerhebliches Gewicht hat. Damit ergibt sich, 
dass doch mehrere Bullen als Stierväter genommen werden, ein Teil wegen des guten Milchzuchtwertes, andere wegen guter Fleischleistung bei akzeptablem Milchzuchtwert, Eine zweite Erklärung für die relativ geringen Verwandtschaftszuwachsraten bei Fleckvieh liegt in der relativ geringen Zentralisierung - es sind mehrere Besamungsstationen und vor allem auch mehrere Zuchtverbände aktiv, d.h. mit Einstellung von Prüfstieren und Selektion bei diesen befasst und vor allem wird im bayerischen Zuchtprogramm die Verwendung von 8 bis 12 Elitestieren verlangt. Auch wird dort vorgeschlagen, dass etwa 10 Jungstiere aus auswärtigen Fleckviehverbänden jährlich geprüft werden.

Bei Tiroler Grauvieh, obzwar zahlenmäßig um vieles kleiner, wird ebenfalls eine Mindestzahl von Stiervätern angestrebt und die beiden Populationen in Nord- und in Südtirol führen jede für sich die Selektion von Zuchtstieren durch. Es ist hier möglich, dass die in den letzten ein oder zwei Jahrzehnten verstärkte Selektion auf Milchleistung auch durch BLUP zu einer stärkeren Betonung herausragender Vererber geführt hat und damit, nicht unähnlich dem Vorgang bei den großen Milchrassen, zu etwas stärkerer Zunahme der Verwandtschaft in der Rasse, was sich in gesteigertem Inzuchtzuwachs niederschlägt.

\section{Danksagung}

Für die zur Verfügung gestellten Daten zur Schätzung von Genfrequenzen bin ich U. HERTNER und O. HAUSEGGER, zu Dank verpflichtet und für Kommentare zum Manuskript A. GOTTSCHALK.

\section{Literatur}

BIJEMA, P.; WOOLLIAMS, J.A.:

Prediction of rates of inbreeding in populations selected on Best Linear Unbiased Predictions of breeding value. Genetics 156 (2000), 361-373

BUSCHMANN, H.:

Blutgruppengenetische Untersuchungen an süddeutschen Rinderrassen. Z. Tierz. Züchtungsbiol. 78 (1962), 12-24

CASANOVA, L.; HAGGER, C.; KÜNZI, N.; SCHNEEBERGER, M.:

Inbreeding in Swiss Braunvieh and its influence on breeding values predicted from a repeatability model. J. Dairy Sci. 75 (1992), 1114-1126

CROW, J.F.; KIMURA, M.:

An introduction to population genetics theory. Harper \& Row, London and NewYork. 1970

ERLACHER, J.:

Blutgruppen und biochemischer Polymorphismus beim Tiroler Grauvieh. Tierärztl. Hochschule Wien, Diss., 1970

FEHLINGS, R.; GRUNDLER, C.; WAUER, A.; PIRCHNER, F.: Inzucht- und Verwandtschaftsverhältnisse in bayerischen Pferderassen (Haflinger, Süddeutsches Kaltblut, Traber). Z. Tierz. Züchtungsbiologie 100 (1983), 81-86

GIBSON, J.P.; KENNEDY, B.W.:

The use of constrained selection indexes in breeding for economic merit. Theor. Appl. Genet. 80 (1990), 801-805

GODDARD, M.E.; WIGGANS, G.R.:

Genetic improvement in dairy cattle. Ch.18 in The genetics of cattle. R. FRIES, A. RUVINSKY, ed. CABI Publishing NewYork. 1999

GRAML, R.; BUCHBERGER, J.; KIRCHMEIER, O.; KIERMEIER, F.; PIRCHNER, F.:

Genfrequenzschätzungen bei Milchproteinen des bayerischen Fleckviehs. Züchtungskunde 56 (1984), 73-87

GRAML, R.; LANG, G.; SCHMID, D.O.; WITTKOWSKI, G.; KRAUSE, I.; BUCHBERGER, J.: 
Schätzung von Inzuchtzuwachs und effektiver Populationsgröße des Murnau-Werdenfelser Rindes und Deutschen Fleckviehs aus Markergenhäufigkeiten. Züchtungskunde 60 (1988), 288-299

GRUNDY, B.; CABALLERO, A.; SANTIAGO, E.; HILL, W.G.:

A note on using biased parameter values and nonrandom mating to reduce rates of inbreeding in selection programmes. Anim. Prod. 59 (1994), 465-468

HATTEMER, H.H.:

Genetic distance between populations. Part 3: Wahlund`s principle as related to genetic distance, and application. Theor. Appl. Genet. 62 (1982), 219-223

HAUSEGGER, O.:

1979, 1998, persönliche Mitteilung

HERTNER, U.:

persönliche Mitteilung, 2001

HIERL, H.F.; ERHARD, L.; OPPELT, E.; SCHMID, D.O.; LÖBHARD, R.:

Untersuchungen der Genfrequenzen biochemischer Merkmale und Blutgruppenloci an bayerischen Rinderrassen. Züchtungskunde 50 (1978), 181-192

HILL, W.G.:

A note on effective population size with overlapping generations. Genetics 92 (1979), 317-321

KERLER, M.:

Die effektive Populationsgröße des Bayerischen Fleckviehs. Diplomarbeit, Inst. Tierzucht, TUM, Weihenstephan. 1987

KROGMEIER, D.; AUMANN, J.; AVERDUNK, G.:

Untersuchungen zur Inzucht in der Gelbvieh- und Braunviehpopulation in Süddeutschland.

LANG, G.: Züchtungskunde 69 (1997), 233-243

Schätzung von Inzuchtzuwachs und effektiver Populationsgröße anhand von Genfrequenzen der Blutgruppen und Milchproteine beim Murnau-Werdenfelser Rind. Diplomarbeit, Inst. Tierzucht, TUM, Weihenstephan, 1987

LATTER, B.D.H.:

Genetic sampling in a random mating central population of constant size and sex ratio. Aust. J. Biol. Sci. 12 (1959), 500-505

LOPEZ-FANJUL, C.:

Selection from crossbreed populations. ABA 42 (1974) 9, 403-416
MEYER, H.:

Verbreitung der a s1- und ß-Caseintypen in deutschen Rinderrassen. Dtsch. Tierärztl. Wochenschrift 74 (1967), 535-537

MEUWISSEN, T.H.E.; WOOLLIAMS, J.A.:

Effective sizes of livestock populations to prevent a decline in fitness. Theor. App. Genet. 89 (1994), 1019-1026

MILLER, L.M.; KAPUSCINSKI, A.R.:

Historical analysis, of genetic variation reveals low effective population size in a Northern Pike population. Genetics 147 (1997), 1249-1258

PIRCHNER, F.:

Population Genetics in Animal Breeding. $2^{\text {nd }}$ ed. Plenum Press, NewYork, POLLAK, E., 1983. A new method for estimating the effective population size from allel frequency changes. Genetics 104 (1983), 531-548

POLLAK, E.:

A new method for estimating the effective population size from allele frequency changes. Genetics 104 (1983), 531-548

REINHARDT, F.: Persönliche Mitteilung, 1986

SCHÜTTE, P.; THOLEN, E.; STORK, F.J.; TRAPPMANN, W.:

Strategien zur Reduzierung des Inzuchtzuwachses in kleinen Basispopulationen der nordrheinwestfälischen Schweineherdbuchzucht. Züchtungskunde 74 (2002), 121-134

SENOU, M.:

Untersuchungen über die Entwicklung der additiv-genetischen Varianz und der Inzucht in geschlossenen Nukleuszuchtprogrammen. TUM Weihenstephan, Diss., 1990

SÖLKNER, J.; FILIPCIC, L.; HAMPSHIRE, N.:

Genetic variability of populations and similarity of subpopulations in Austrian cattle breeds determined by the analysis of pedigrees. Anim. Sci. 67 (1998), 249-256

TEBRAKE, M.F.H.; GROEN, A.F.; VAN DER LUGT, A.W.:

Trends in inbreeding in Dutch Black and White cattle. J. Anim. Breed. Genet. 111 (1994), 356-366

WAPLES, R.S.: 
A generalized approach for estimating effective population size from temporal changes in allele frequency. Genetics 121 (1989), 379-391

WRAY, N.R.; THOMPSON, R.:

Prediction of rates of inbreeding in selected populations. Genet. Res. 55 (1990), 41-54

Eingegangen: 11.04.2002

Akzeptiert: 20.06.2002

Anschrift des Verfassers

Prof. Drs. h. c. FRANZ PIRCHNER, PhD

Mosengasse 4

A - 6020 Innsbruck

Austria 